\title{
Diffusion Behavior of Methane in 3D Kerogen Models
}

Kai Bin Yu ${ }^{\dagger}$, Geoffrey M. Bowers ${ }^{\ddagger}$, Narasimhan Loganathan ${ }^{\S}$, Andrey G. Kalinichev^ and A. Ozgur Yazaydin ${ }^{\dagger},{ }^{*}$

'Department of Chemical Engineering, University College London, London WC1E 7JE, United Kingdom

tDepartment of Chemistry and Biochemistry, St. Mary's College of Maryland, St. Mary's City, Maryland 20686, USA

§Department of Chemistry, Michigan State University, East Lansing, Michigan 48824, USA

${ }^{\wedge}$ Laboratoire SUBATECH (UMR 6457 - Institut Mines-Télécom Atlantique, Université de Nantes, CNRS/IN2P3), Nantes, France

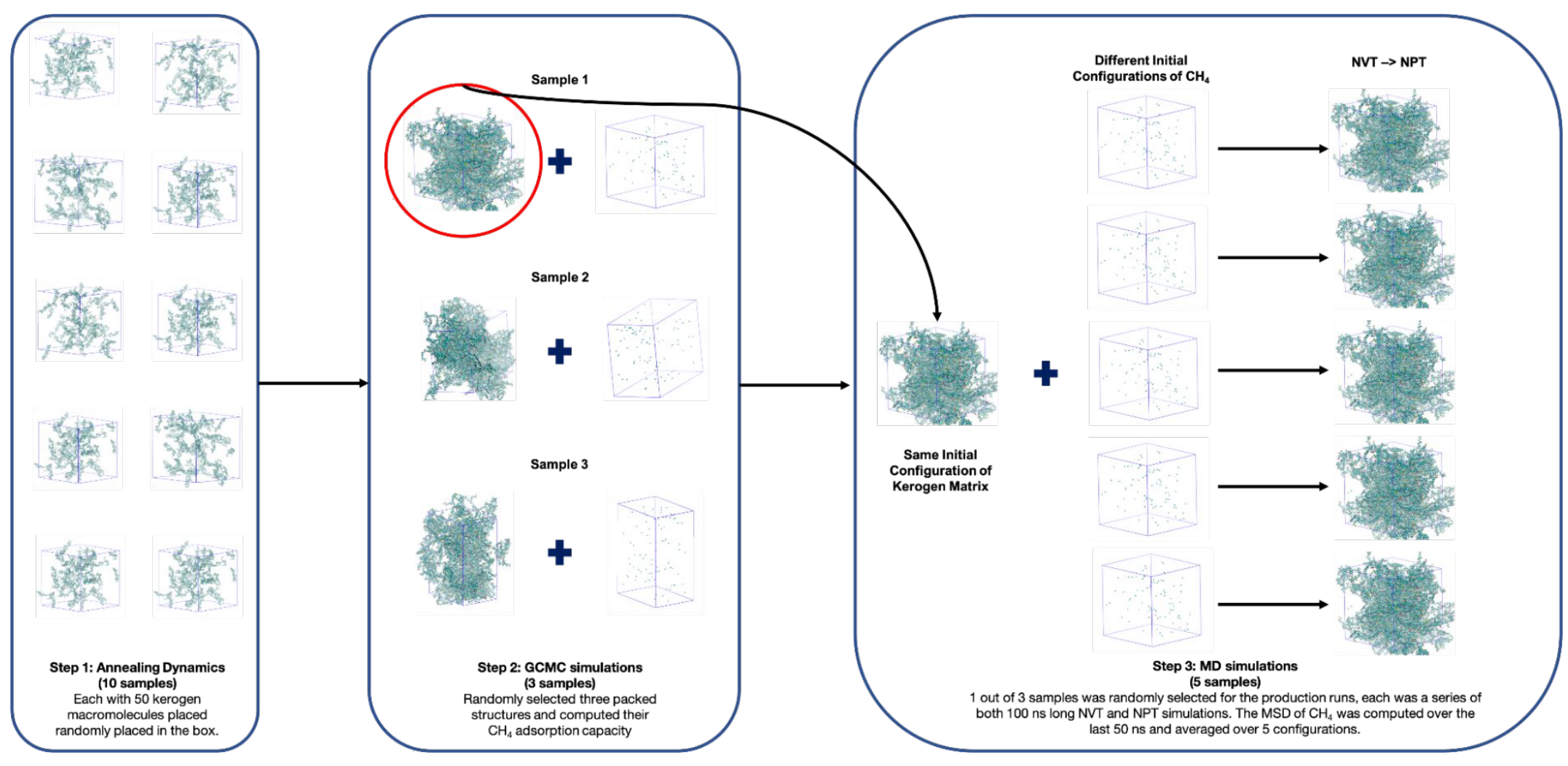


Figure S1: A workflow diagram of the simulation set up procedure. 
(A) Type I-A

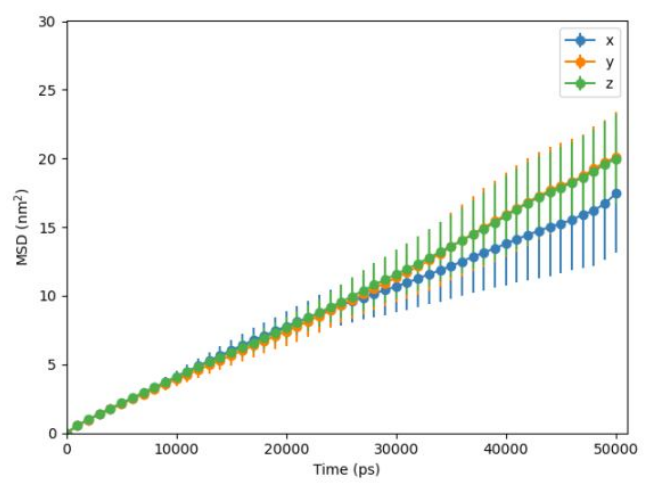

(C) Type II-B

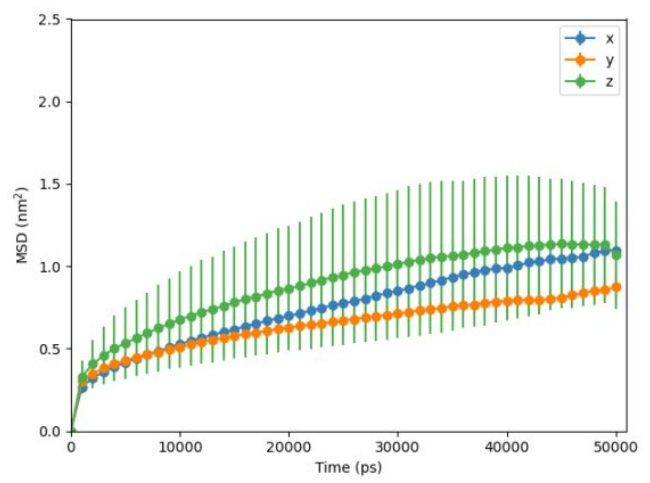

(E) Type II-D

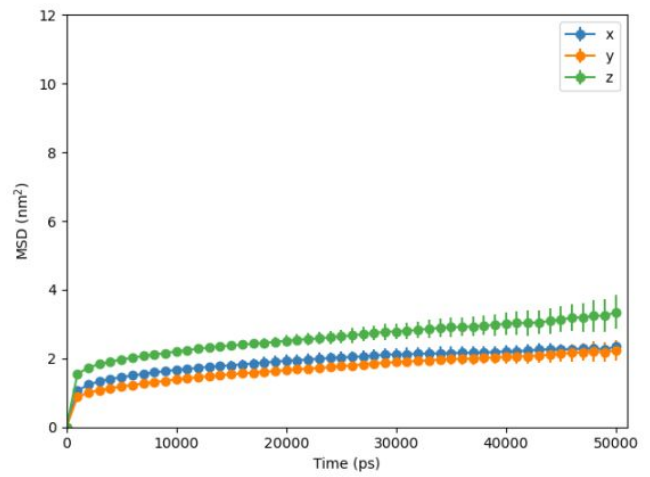

(B) Type II-A

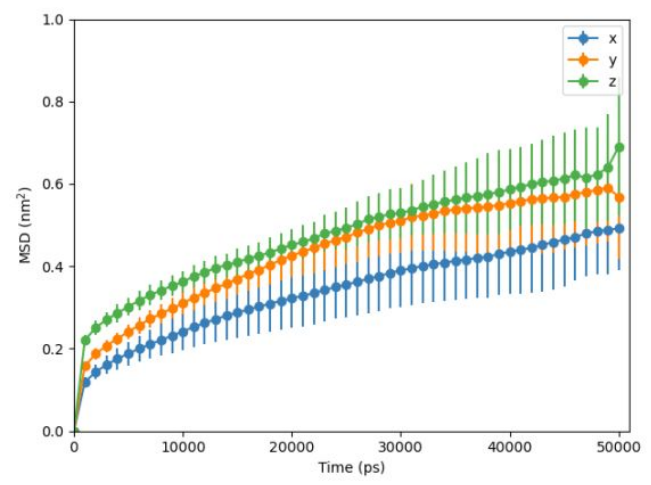

(D) Type II-C

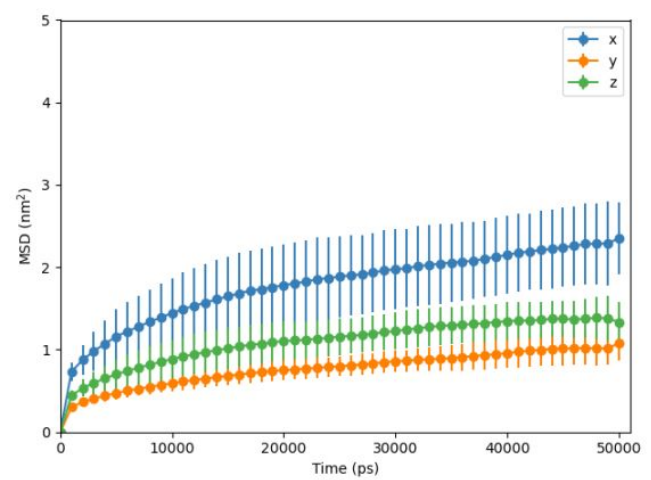

(F) Type III-A

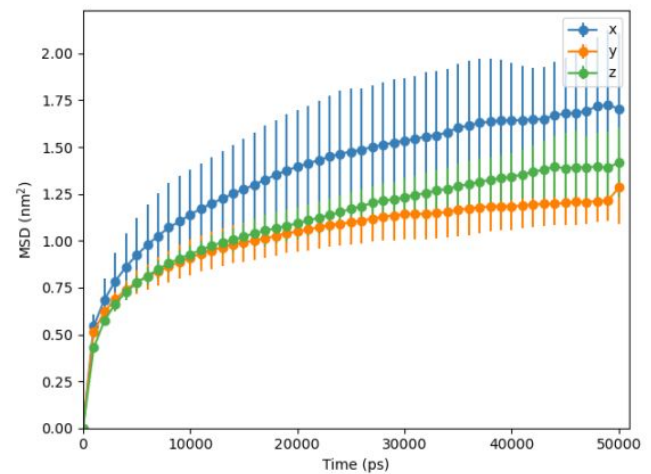

Figure $\mathrm{S} 2$ : The average MSD of $\mathrm{CH}_{4}$ in the $\mathrm{x}, \mathrm{y}$ and $\mathrm{z}$ directions in six kerogen types in NVT ensemble at $365 \mathrm{~K}$. 
(A) Type I-A

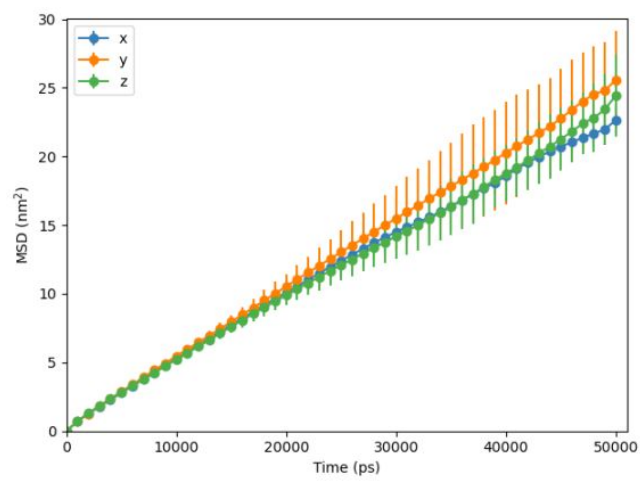

(C) Type II-B

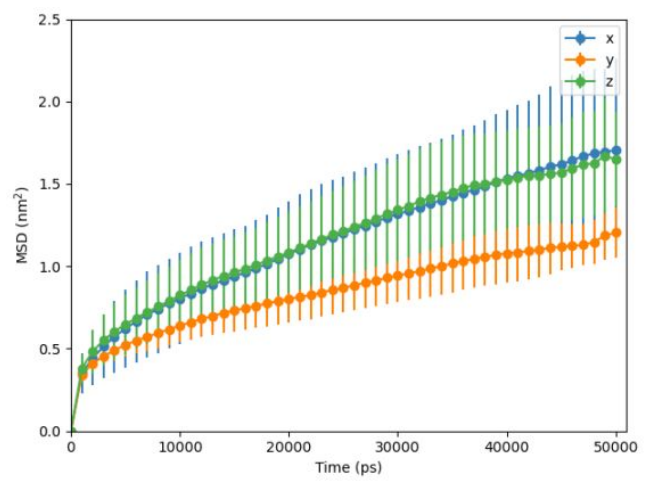

(E) Type II-D

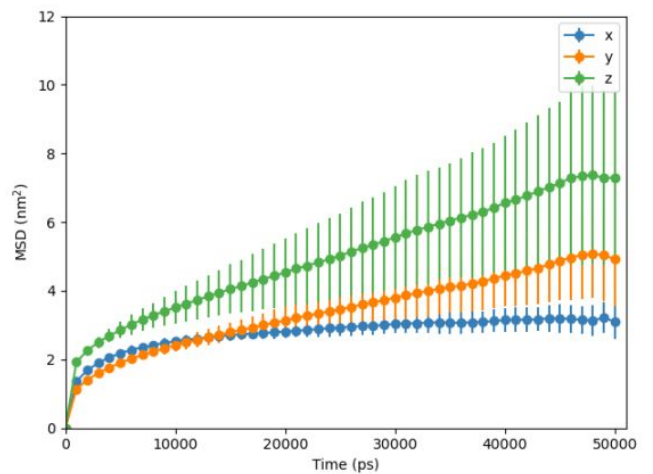

(B) Type II-A

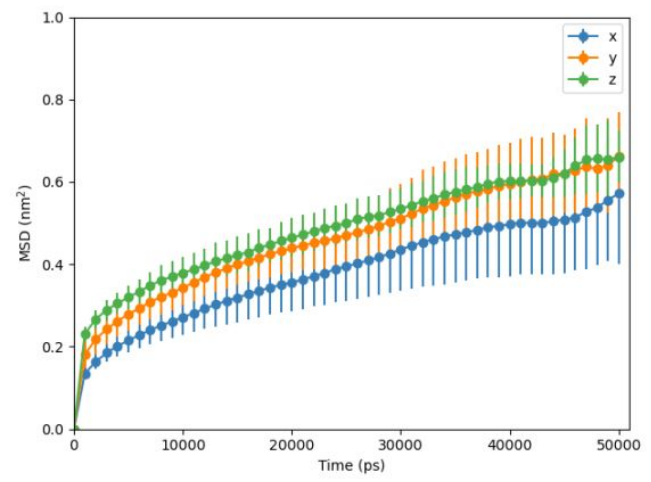

(D) Type II-C

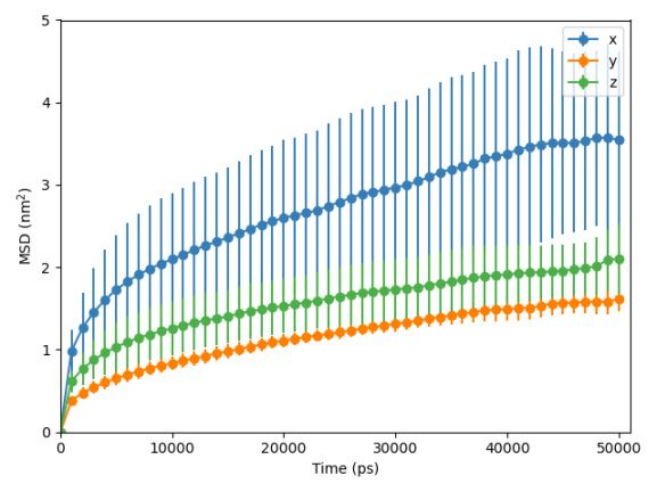

(F) Type III-A

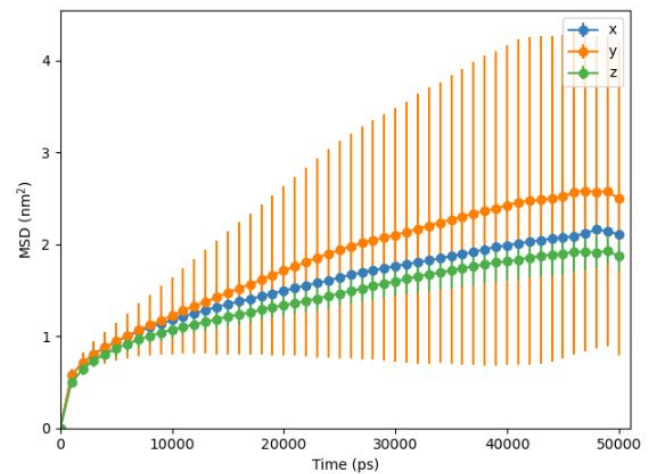

Figure S3: The average MSD of $\mathrm{CH}_{4}$ in the $\mathrm{x}, \mathrm{y}$ and $\mathrm{z}$ directions in six kerogen types in NPT ensemble at $365 \mathrm{~K}$ and 275 bar. 
(A) Type I-A

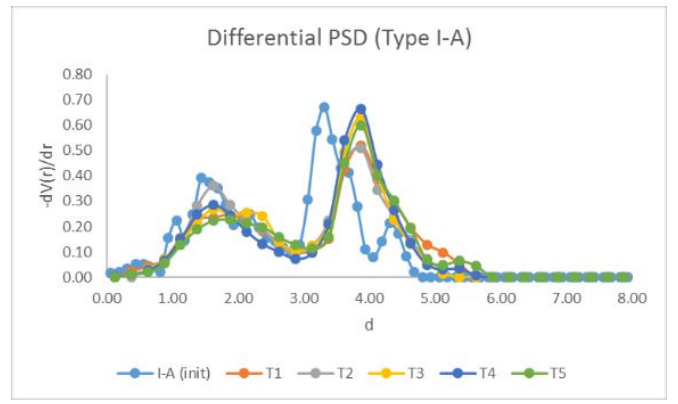

(C) Type II-B

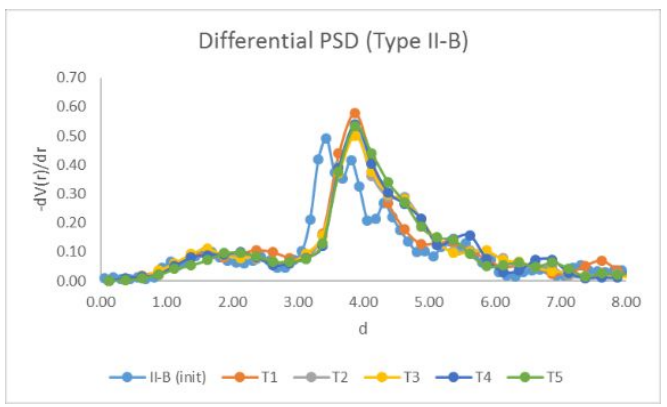

(E) Type II-D

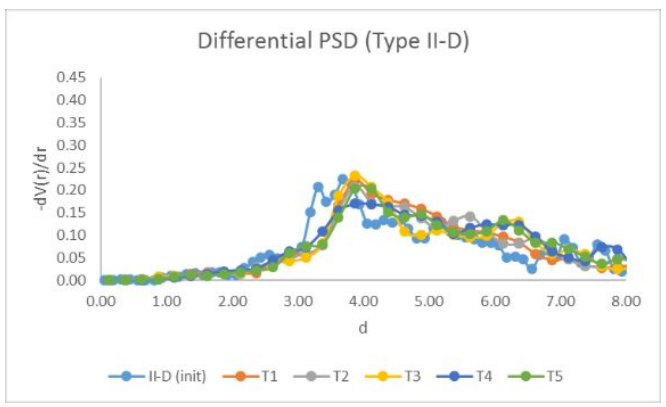

(B) Type II-A

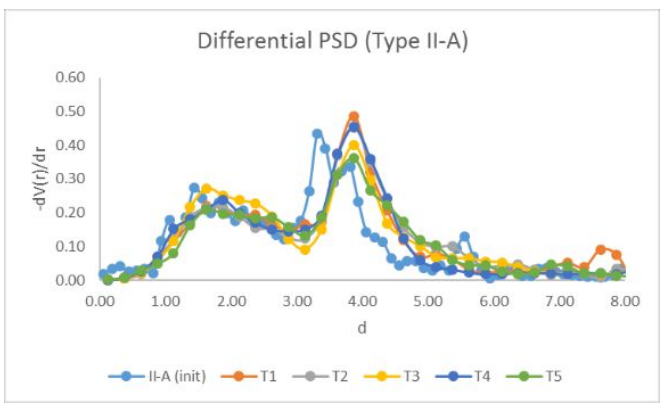

(D) Type II-C

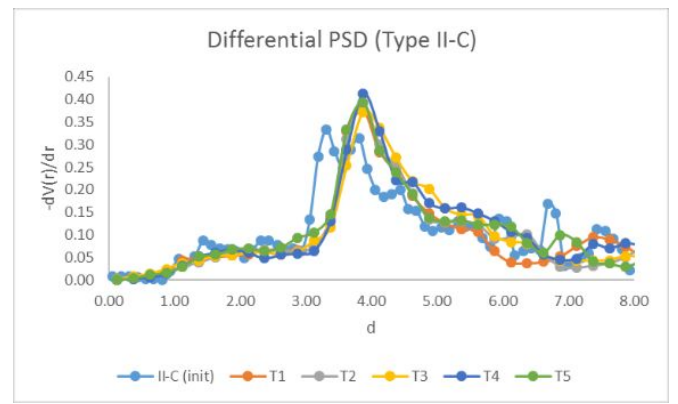

(F) Type III-A

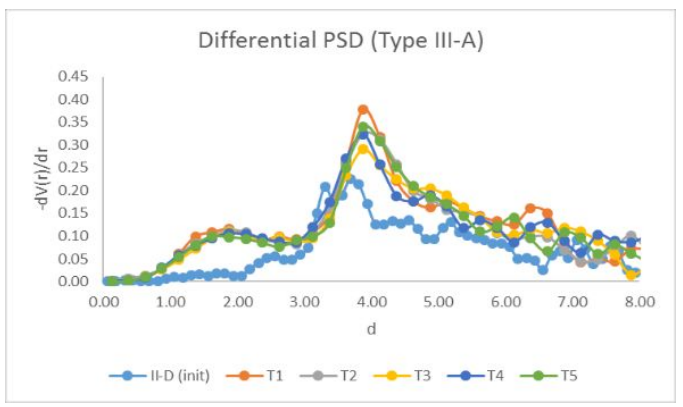

Figure S4: Pore size distributions of kerogens, before (initial) and after methane loading in five independent runs (T1, T2, T3, T4, and T5). 
(A) Type I-A

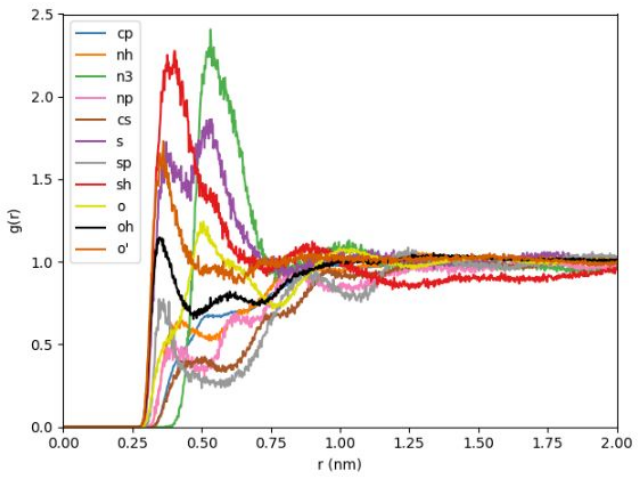

(C) Type II-B

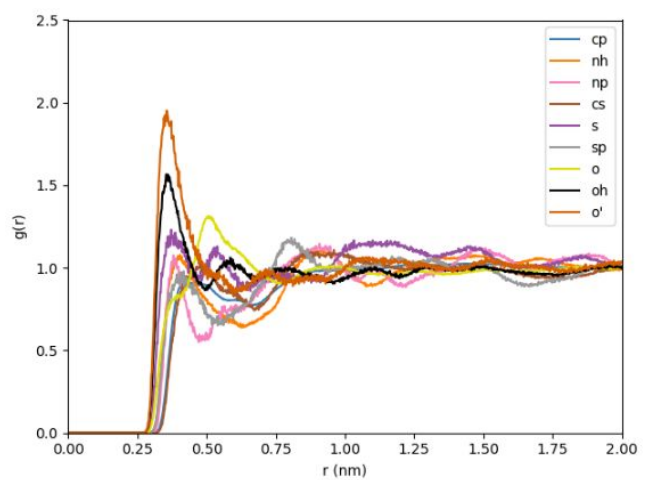

(E) Type II-D

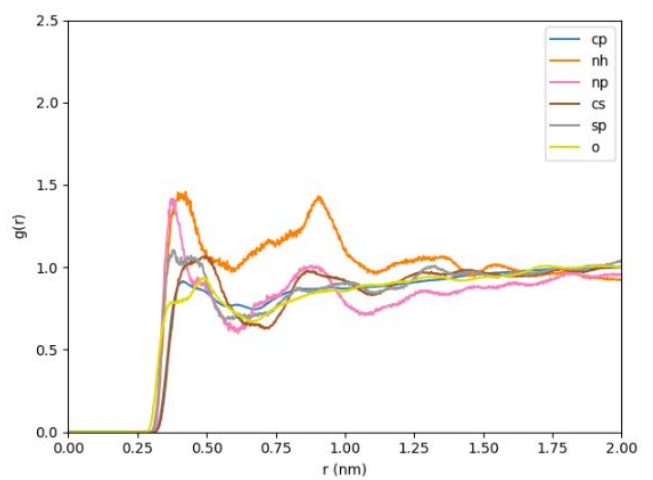

(B) Type II-A

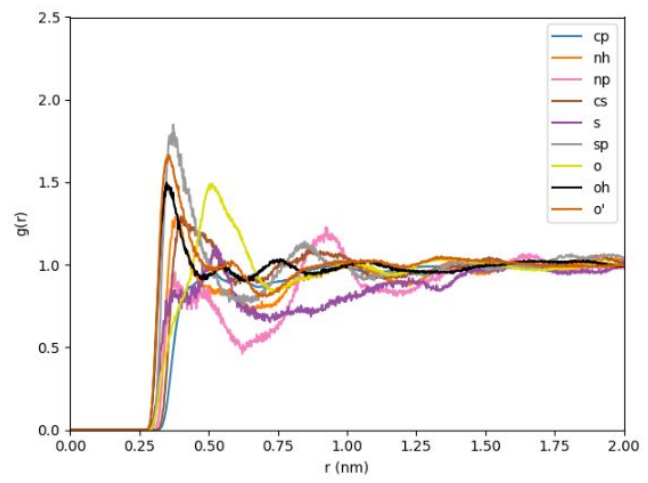

(D) Type II-C

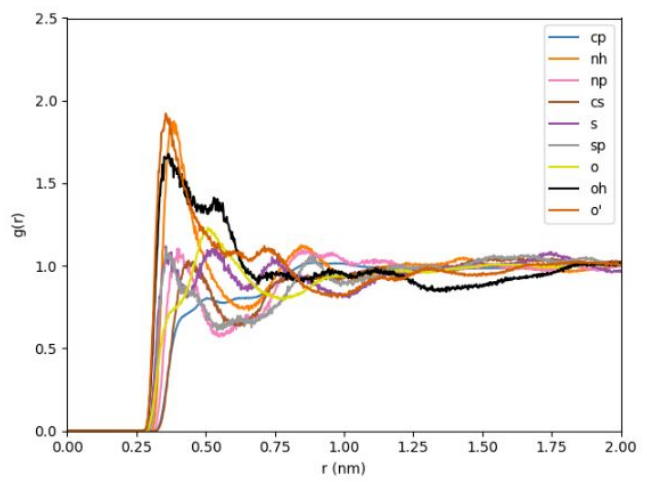

(F) Type III-A

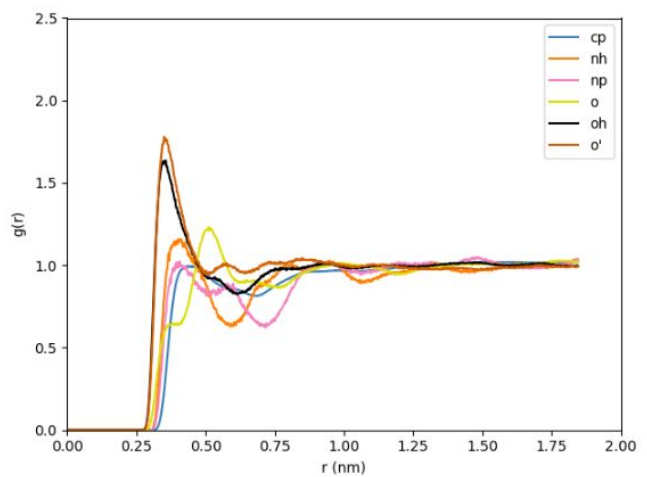

Figure S5: RDFs between $\mathrm{CH}_{4}$ and atoms of different functional groups in kerogen macromolecules of various types at $365 \mathrm{~K}$ and 275 bar obtained from NPT MD simulations. Atom types defined by the CVFF force field and included in the above RDF plots are as follows: (cp) $\mathrm{sp} 2$ aromatic carbon, (cs) sp2 carbon involved in thiophene, (nh) sp2 nitrogen in 5-or 6- membered ring with hydrogen attached, (np) sp2 aromatic nitrogen (partial double bonds), (n3) sp3 nitrogen with three substituents, (o) sp3 oxygen in ether or ester groups, (oh) oxygen in hydroxyl $(\mathrm{OH})$ group, (o') oxygen in carbonyl $(\mathrm{C}=\mathrm{O})$ group, (s) sulfur in methionine (C-S-C) group, (sh) sulfur in sulfhydryl (-SH) group, and (sp) sulfur in thiophene. 
(A) Type I-A

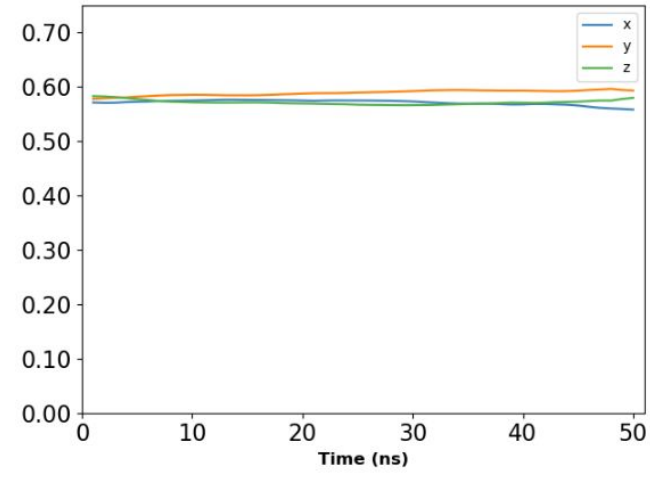

(C) Type II-B

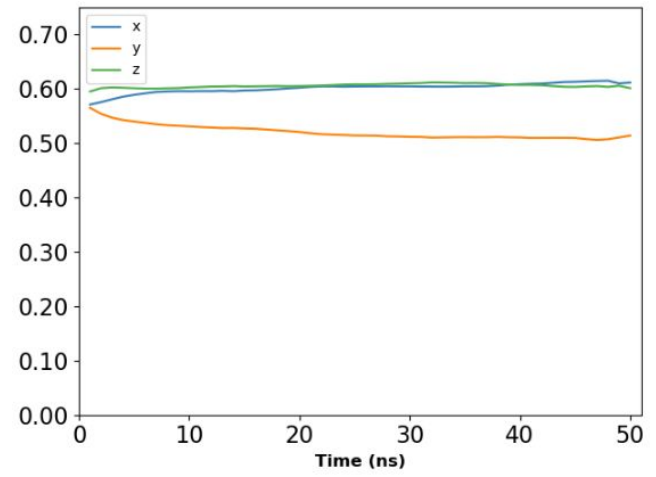

(E) Type II-D

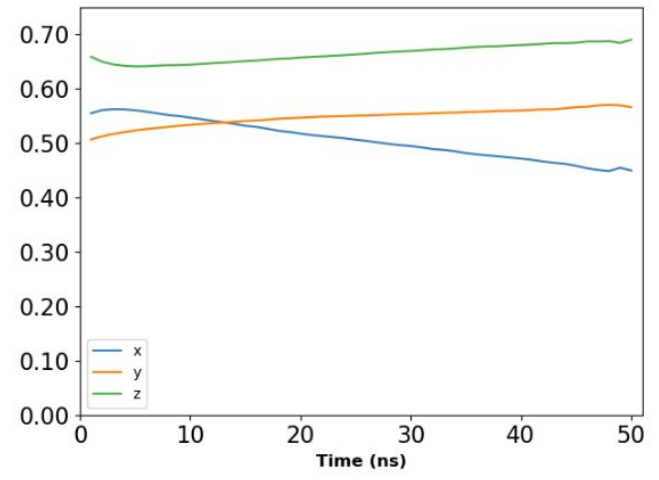

(B) Type II-A

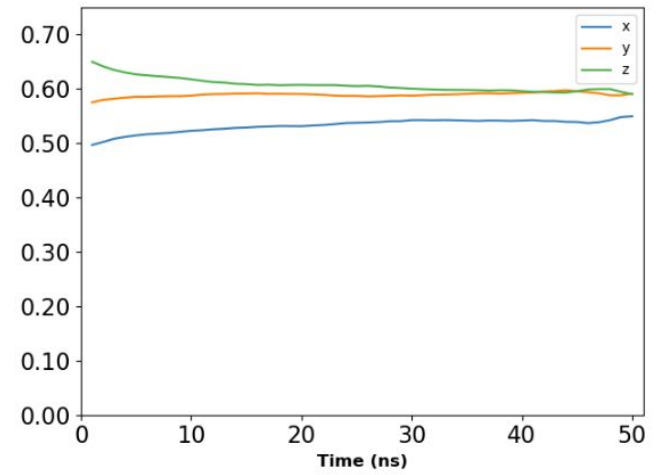

(D) Type II-C

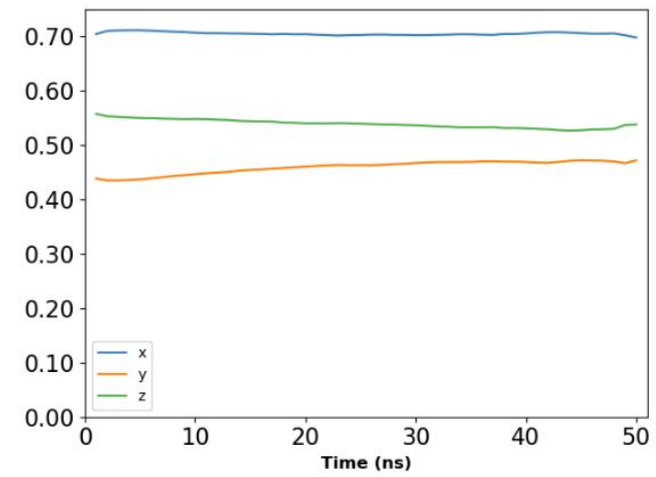

(F) Type III-A

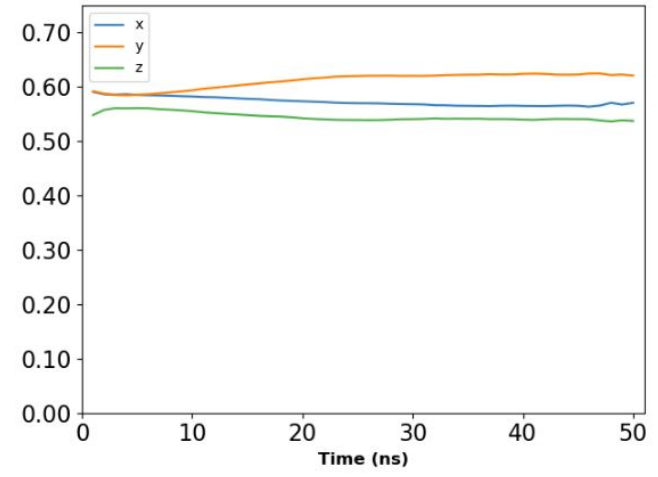

Figure S6: Normalized MSD over time using the formula $\sqrt{\left\langle i^{2}\right\rangle} / \sqrt{\left\langle x^{2}\right\rangle+\left\langle y^{2}\right\rangle+\left\langle z^{2}\right\rangle}$, where $i$ is one of the three directions on the Cartesian space. 